\title{
COMPLEX PARALLISABLE MANIFOLDS
}

\section{HSIEN-CHUNG WANG}

1. Introduction. Let $M$ be a complex manifold of $2 n$ dimension. It will be called complex parallisable if there exist, over $M, n$ holomorphic vector fields linearly independent everywhere. We see immediately that this is the natural counterpart, in the theory of complex manifolds, of our ordinary completely parallisable spaces. It is the aim of the present paper to study the compact complex parallisable manifolds. Unlike the real analytic case, we find that they can never be simply-connected and they can be regarded, up to a holomorphic homeomorphism, as the compact coset spaces of complex Lie groups with discrete isotropic subgroup. Such manifolds are not Kählerian in general. They are Kählerian if and only if they are the complex tori. By using theory of Lie groups, we can construct numerous such manifolds other than tori. ${ }^{1}$ For example, given any finitely generated nilpotent abstract group $D_{1}$ without periodic element, there exists a compact complex parallisable manifold whose fundamental group contains $D_{1}$ as a subgroup.

The idea of the proof of our results is extremely simple. In fact, each complex parallisable manifold $M$ has $n$ holomorphic 1 -forms $\omega^{\alpha}$ linearly independent everywhere. If $M$ is compact, then the $\omega$ 's satisfy the so-called Maurer-Cartan equations, and then define a complex Lie group structure over the universal covering space of $M$. It follows then that $M$ is a homogeneous space of this complex Lie group with discrete isotropic subgroup. Of course, taking account of a theorem of Bochner-Montgomergy [1], we can get the same result by using only the vector fields. Here, the differential form arguments are adopted simply because this approach gives us, in a natural manner, the number of linearly independent abelian differentials of the first kind over $M$. This information facilitates the determination of the condition for $M$ to be Kählerian.

2. Holomorphic 1-forms over a compact parallisable manifold. Let $M$ be a complex manifold of $2 n$ dimension. Just the same as in the case of real differential manifolds [4, p. 22], we can define the bundle $B$ of complex tensors, over $M$, of a given type. This bundle is a

Presented to the Society, December 28, 1952; received by the editors December 18, 1953.

${ }^{1}$ Such an example was first given by K. Iwasawa to exhibit certain properties of non-Kählerian, compact complex manifolds. 
complex bundle in the sense that all the spaces and mappings involved in the bundle structure are complex analytic. The fibre at each point $x$ of $M$ is a linear space over the complex numbers. By a complex tensor field $X$, we mean a cross section of a complex tensor bundle $B$. The tensor field $X$ is called holomorphic if the cross section $X: M \rightarrow B$ is a holomorphic mapping. In case that $B$ is the bundle of skew symmetric covariant tensors of order $p$, we shall call the corresponding tensor field a $p$-form.

We know that the complex tangent bundle and the bundle of complex covariant vectors are dual to each other. Therefore, for each complex tangent vector field $X$ and each complex 1 -form $\omega$, the product $\omega X$ is defined and is a complex-valued function over $M$. In case that both $X$ and $\omega$ are holomorphic, their product $\omega X$ is a holomorphic function of $M$.

From now on, we assume $M$ to be compact and complex parallisable, i.e., there exist $n$ holomorphic tangent vector fields $X_{\alpha}$ $(\alpha=1,2, \cdots, n)$ such that, at each point $x$, the $n$ vectors $X_{1}(x)$, $X_{2}(x), \cdots, X_{n}(x)$ are linearly independent. Thus the complex tangent space $T_{x}$ of $M$ at $x$ is spanned by $X_{\alpha}(x)$. It follows then that there are $n$ holomorphic 1 -forms $\omega^{\beta}$ satisfying the equations

$$
\omega^{\beta} X_{\alpha}=\delta_{\alpha}^{\beta} \quad(\alpha, \beta=1,2, \cdots, n)
$$

where $\delta_{\alpha}^{\beta}$ denote the Kronecker deltas, or more precisely, the constant functions over $M$ with the value $\delta_{\alpha}^{\beta}$. These 1 -forms $\omega^{\alpha}$ are uniquely determined by (1) and are linearly independent everywhere. In terms of local coordinates, the expressions for $\omega^{\alpha}$ can be written out explicitly. Let $U$ be a coordinate neighborhood with coordinates $z^{1}, z^{2}, \cdots, z^{n}$. Then $X_{\alpha}$ is represented by $n$ holomorphic functions $X_{\alpha}^{1}(z), X_{\alpha}^{2}(z), \cdots, X_{\alpha}^{n}(z)$ in the variables $z^{1}, z^{2}, \cdots, z^{n}$. From our assumption, the determinant $\left|X_{\alpha}^{t}(z)\right|$ never vanishes, and thus the matrix $\left(X_{\alpha}^{i}(z)\right)$ has an inverse, say $\left(f_{i}^{\alpha}(z)\right)$. The 1 -form $\sum_{i} f_{i}^{\alpha}(z) d z^{i}$ is our $\omega^{\alpha}$ in terms of the local coordinates $z^{i}$.

The forms $\omega^{\alpha}$ being holomorphic and linearly independent everywhere, their exterior products $\omega^{\alpha} \wedge \omega^{\beta}(\alpha<\beta)$ must also be holomorphic and linearly independent everywhere. Let $S_{x}$ be the space of all skew symmetric covariant tensors of order 2 at $x$. Since $S_{x}$ is of dimension $n(n-1) / 2,\left(\omega^{\alpha} \wedge \omega^{\beta}\right)(x)(\alpha<\beta)$ form a basis of $S_{x}$. The exterior derivative $d \omega^{\gamma}$ is a 2 -form. Thus $\left(d \omega^{\gamma}\right)(x)$ is a linear combination of $\left(\omega^{\alpha} \wedge \omega^{\beta}\right)(x)(\alpha<\beta)$ with complex coefficients:

$$
d \omega^{\gamma}(x)=\sum_{\alpha<\beta} C_{\alpha \beta}^{\gamma}(x)\left(\omega^{\alpha} \wedge \omega^{\beta}\right)(x)
$$


Since the complex numbers $C_{\alpha \beta}^{\gamma}(x)$ are uniquely determined by the point $x$, we can regard $x \rightarrow C_{\alpha \beta}^{\gamma}(x)$ as complex-valued functions over $M$. We know that $d \omega^{\gamma}, \omega^{\alpha} \wedge \omega^{\beta}$ are holomorphic so that $C_{\alpha \beta}^{\gamma}(x)$ are holomorphic functions over $M$. But $M$ is compact. Thus $C_{\alpha \beta}^{\gamma}(x)$ must be constant functions, and thus $C_{\alpha \beta}^{\gamma}(x)=C_{\alpha \beta}^{\gamma}$. By putting $C_{\alpha \beta}^{\gamma}=-C_{\beta \alpha}^{\gamma}$, $\alpha<\beta$, and $C_{\beta \beta}^{\gamma}=0$, we can write (2) in a better form:

$$
d \omega^{\gamma}=\frac{1}{2} \sum_{\alpha, \beta} C_{\alpha \beta}^{\gamma} \omega^{\alpha} \wedge \omega^{\beta},
$$

where

$$
C_{\alpha \beta}^{\gamma}+C_{\beta \alpha}^{\gamma}=0,
$$$$
C_{\alpha \beta}^{\gamma}=\text { constants. }
$$

Moreover, from the fact $d d \omega^{\gamma}=0$, it follows that

$$
\sum_{\epsilon}\left(C_{\alpha \beta}^{\epsilon} C_{\epsilon \gamma}^{\delta}+C_{\beta \gamma}^{\epsilon} C_{\epsilon \alpha}^{\delta}+C_{\gamma \alpha}^{\epsilon} C_{\epsilon \beta}^{\delta}\right)=0 .
$$

3. Structure of a compact complex parallisable manifold. We see at once from (3), (4), (5) that the forms $\omega^{\alpha}$ define a local Lie group. However, this local Lie group, in general, cannot be extended to the entire space. For this reason, we consider the universal covering space $G$ of $M . G$ inherits a complex structure in the natural manner. Let $\phi: G \rightarrow M$ be the covering map. It induces a homomorphism $\phi^{*}$ of the Grassmann algebra of $M$ into that of $G$. Since $\phi$ is a local homeomorphism, $\phi^{*}$ is one-to-one. Let us denote $\phi^{*}\left(\omega^{\alpha}\right)$ by $\tilde{\omega}^{\alpha}$. These forms $\tilde{\omega}^{\alpha}$ are evidently holomorphic, linearly independent everywhere, and

$$
d \tilde{\omega}^{\gamma}=\frac{1}{2} \sum_{\alpha, \beta} C_{\alpha \beta}^{\gamma} \tilde{\omega}^{\alpha} \wedge \tilde{\omega}^{\beta}
$$

where the constants $C_{\alpha \beta}^{\gamma}$ are subject to (4) and (5). The quadratic differential form

$$
d s^{2}=\sum_{\alpha} \tilde{\omega}^{\alpha} \overline{\tilde{\omega}}^{\alpha}
$$

defines over $G$ a nondegenerate Hermitian metric (in general, not Kählerian). We shall show that $G$ is complete with respect to this metric in the sense of Riemannian geometry. ${ }^{2}$ For this purpose, let us consider the manifold $M$ with the Hermitian metric $\sum \omega^{\alpha} \bar{\omega}^{\alpha}$. Since $M$ is compact, $M$ is, of course, complete with respect to this metric.

2 A Riemannian space is called complete if there exists a positive number $\eta$ such that, given any point $p$ and any direction at $p$, we can construct a geodesic arc, issued from $p$, of length $\eta$ which is tangent to the given direction at $p$. 
From the fact that

$$
\phi^{*}\left(\sum \omega^{\alpha} \bar{\omega}^{\alpha}\right)=\sum \tilde{\omega}^{\alpha} \overline{\tilde{\omega}}^{\alpha},
$$

we see immediately that $\left\{G, d s^{2}\right\}$ is a covering space of $\left\{M, \sum \omega^{\alpha} \bar{\omega}^{\alpha}\right\}$ not only in the sense of complex manifolds but also in the sense of Hermitian geometry. Thus the completeness of $M$ implies the completeness of $\left\{G, d s^{2}\right\}$.

Now we have, over $G, n$ holomorphic 1 -forms $\tilde{\omega}^{\alpha}$ which are everywhere linearly independent and satisfy the Maurer-Cartan equation (6). Moreover, $G$ is simply-connected and complete with respect to the metric

$$
d s^{2}=\sum \tilde{\omega}^{\alpha} \overline{\tilde{\omega}}^{\alpha} .
$$

It is known that [2, pp. 188-192], under these conditions, $G$ can be given a group structure such that the multiplication is holomorphic and that all the left translations leave invariant the forms $\tilde{\omega}^{\alpha}$. Thus we know that $G$ is a complex Lie group with $\tilde{\omega}^{\alpha}$ as its Maurer-Cartan forms. Let $D$ be the group of covering transformations of $G$ which gives the space $M$. For each $\sigma \in D$, we have $\phi \sigma=\phi$. It follows that $\sigma^{*} \phi^{*}=\phi^{*}$, and

$$
\tilde{\omega}^{\alpha}=\phi^{*}\left(\omega^{\alpha}\right)=\sigma^{*} \phi^{*}\left(\omega^{\alpha}\right)=\sigma^{*}\left(\tilde{\omega}^{\alpha}\right)
$$

where $\sigma^{*}$ denotes the isomorphism of the Grassmann algebra of $G$ onto itself induced by $\sigma$. In other words, each covering transformation in $D$ leaves unaltered the Maurer-Cartan forms $\bar{\omega}^{\alpha}$. In the theory of Lie groups, it is well known that such a transformation must be a left translation of the group. Thus we can identify $D$ with a subgroup of $G$. This identification gives a complex analytic homeomorphism between $M$ and the space $G / D$ of right cosets. We have therefore proved

Theorem 1. Let $M$ be a compact, complex parallisable manifold. Then $M$ is complex analytically homeomorphic with a complex coset space $^{3} G / D$ of a complex Lie group $G$ over a discrete subgroup $D$.

It is evident that each coset space of a complex Lie group over a discrete subgroup is complex parallisable. But we should note that Theorem I no longer holds for noncompact $M$. The bounded domains serve as the simplest counter-examples.

4. Holomorphic cohomology ring of $M$. Let $E$ be a complex manifold, and $\mathfrak{U}(E)$ the Grassmann algebra of all holomorphic differential

\footnotetext{
${ }^{3} G / D$ inherits a complex structure from $G$. For precise definition, cf. [5].
} 
forms over $E$. We know that $\mathfrak{U}(E)$ is a graded algebra with the exterior derivation $d$ as the differential operator. Just the same as in the ordinary de Rham theory for real differential manifolds, we can define a cohomology ring $C(E)=\sum_{i=1}^{n} C^{i}(E)$ out of $\mathfrak{U}(E)$. Let us call $C(E)$ the holomorphic cohomology ring, and $C^{i}(E)$ the $i$-dimensional holomorphic cohomology group of $E$. This $C(E)$ has relations with our ordinary cohomology ring of $E$. For example, if $E$ is compact and Kählerian, then $b^{i} \geqq 2 \operatorname{dim} C^{i}(E), i>1, b^{1}=2 \operatorname{dim} C^{1}(E)$, where $b^{i}$ denotes the $i$ th Betti-number of $E$. In case that $E$ can be imbedded regularly in a complex Euclidean space as a submanifold, then $b^{i}$ $=\operatorname{dim} C^{i}(E)$.

Now let us confine ourselves to the compact, complex parallisable manifold. We assume that the notations $G, \omega^{\alpha}, \tilde{\omega}^{\alpha}, \phi, \phi^{*}$ have the same meaning as in $\$ \S 2,3$. Since $\omega^{\alpha}$ are everywhere linearly independent over $M$, each holomorphic $p$-form $\Omega$ can be written, in a unique way, as a sum

$$
\Omega=\sum_{\alpha_{1}<\alpha_{2}<\cdots<\alpha_{p}} C_{\alpha_{1} \alpha_{2} \cdots \alpha_{p}} \omega^{\alpha_{1}} \wedge \omega^{\alpha_{2}} \wedge \cdots \wedge \omega^{\alpha_{p}}
$$

where the $C$ 's are holomorphic functions over $M$. From the compactness of $M$, these functions $C_{\alpha_{1} \ldots \alpha_{p}}$ must be constants. Thus each holomorphic form $\Omega$ is a polynomial of the $\omega$ 's with constant coefficients. It follows then that $\phi^{*}(\Omega)$ is a polynomial in the $\tilde{\omega}^{\prime}$ s with constant coefficients, and hence is a left-invariant complex differential form of $G$. Conversely, we can easily see that if a complex differential form of $G$ is invariant under left translations, then it must be a polynomial of $\tilde{\omega}^{\alpha}$ with constant coefficients. Therefore $\phi^{*}: \mathfrak{U}(M) \rightarrow \mathfrak{B}(G)$ is an isomorphism of $\mathfrak{U}(M)$ onto the Grassmann algebra $\mathfrak{B}(G)$ of all left-invariant complex differential forms of $G$. Here, isomorphism is used in the sense that it is one-to-one, linear, and preserves both the multiplication and the differential operator $d$. Thus the holomorphic cohomology ring $C(E)$ is isomorphic with the cohomology ring $C(\mathfrak{B}(G)$ ) defined by $\mathfrak{B}(G)$. On the other hand, it is known [3] that $C(\mathfrak{B}(G)$ ) coincides with the cohomology ring $C(\mathbb{R})$ of the complex Lie algebra $\mathbb{R}$ of the complex Lie group $G$. Thus we have proved

Theorem 2. Let $M=G / D$ be a compact, complex parallisable manifold represented in the form of coset space $G / D$. Then the holomorphic cohomology ring $C(M)$ of $M$ is isomorphic with the cohomology ring of the complex Lie algebra $\&$ of $G$.

This tells us that $C(M)$ depends only on the local structure of $G$ but not on the uniform discrete subgroup $D$ of $G$. 
5. Consequences of Theorem 2. An abelian differential of the first kind is, by definition, a closed holomorphic 1 -form. Now let $k$ be the number of linearly independent abelian differentials of the first kind over our compact, complex parallisable manifold $M$. Since $M$ is compact, a nonzero closed holomorphic 1 -form can never be a coboundary. Thus $k=\operatorname{dim} C^{1}(M)$. Theorem 2 then implies $k=\operatorname{dim} C^{1}(\mathfrak{R})$. It is well known, in the theory of cohomology rings of Lie algebras, that $\operatorname{dim} C^{1}(\mathfrak{R})=\operatorname{dim}\left(\mathfrak{R} / \mathfrak{R}^{\prime}\right)$ where $\mathfrak{R}^{\prime}$ denotes the derived algebra of $\mathfrak{R}$. Using groups instead of Lie algebras and denoting by $G^{\prime}$ the commutator subgroup of $G$, we have

$$
k=\operatorname{dim} C^{1}(M)=\operatorname{dim}\left(\mathfrak{R} / \mathbb{R}^{\prime}\right)=2^{-1} \operatorname{dim}\left(G / G^{\prime}\right){ }^{4}
$$

We note that $G^{\prime}$ is always closed in $G$ as $G$ is simply-connected. Thus

Corollary 1. Let $k$ be the number of linearly independent abelian differentials of the first kind over the compact, complex parallisable manifold $M=G / D$. Then $2 k=\operatorname{dim} G / G^{\prime}$ where $G^{\prime}$ denotes the commutator subgroup of $G$.

This corollary gives us the following necessary and sufficient condition for $M$ to be Kählerian:

Corollary 2. A compact, complex parallisable manifold $M$ is Kählerian if and only if it is a complex torus.

Proof. Firstly, it is evident that each complex torus is compact, Kählerian, and complex parallisable. Conversely, suppose $M=G / D$ to be Kählerian. Then the holomorphic 1 -forms $\omega^{\alpha}$ must be closed. Thus $M$ has $n$ linearly independent abelian differentials of the first kind where $2 n$ is the dimension of $M$. Corollary 1 then tells us that $\operatorname{dim} G^{\prime}=0$. In other words, $G$ is abelian, and thus $M=G / D$ is the $2 n$ dimensional complex torus.

\section{REFERENCES}

1. S. Bochner and D. Montgomery, Groups on analytic manifolds, Ann. of Math. vol. 48 (1947) pp. 659-669.

2. E. Cartan, La théorie des groupes finis et continus et la géomêtrie différentielle, Paris, 1947.

3. C. Chevalley and S. Eilenberg, Cohomology theory of Lie groups and Lie algebras, Trans. Amer. Math. Soc. vol. 63 (1948) pp. 85-124.

4. N. E. Steenrod, The topology of fibre bundles, Princeton, 1951.

5. H. C. Wang, Closed manifolds with homogeneous complex structure, Amer. J. Math. vol. 76, pp. 1-32.

Alabama Polytechnic Institute

\footnotetext{
- The dimension of a topological group is used in the topological sense.
} 